\title{
Shannon Information in $K$-records for Pareto-type Distributions
}

\author{
Zohreh Zamani \\ Department of Statistics, Faculty of Mathematics and Computer, Shahid Bahonar University of Kerman \\ Kerman, Iran \\ z.zamani.62@gmail.com \\ Mohsen Madadi \\ Department of Statistics, Faculty of Mathematics and Computer, Shahid Bahonar University of Kerman \\ Kerman, Iran \\ mhnmadadi@gmail.com
}

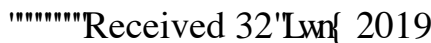

Accepted प००1 RYP EH2017

\begin{abstract}
Pareto distributions provides models for many applications in the social, natural and physical sciences. In this paper, we derive the Shannon information contained in upper (lower) $k$-record values and associated $k$-record times of Pareto-type distributions for a finite sample of fixed size and for an inverse sampling plan. Properties of the Shannon information of the $k$-record values associated with Pareto-type distributions are investigated, both analytically and numerically.
\end{abstract}

Keywords: $K$-record values; $K$-record times; Shannon information; Digamma function; Zeta function; Differential entropy; Record data

2010 Mathematics Subject Classification: 94A17, 54C70, 94A15

\section{Introduction}

The origin of the term entropy goes back to the works of Clausius (1864) and Boltzmann (1872) in thermodynamics. The idea of information-theoretic entropy was first introduced by Shannon (1948). Park (1995), Ebrahimi et al. (2004) and Oluyede (2006) obtained various results on the information properties of order statistics. Yari and Borzadaran (2010) calculated the Shannon entropy for Paretotype distributions and their order statistics. Baratpour et al. (2007) derived some results related to the Shannon entropy and Rényi entropy for record values.

In 1976, Dzubdziela and Kopociński introduced the concept of $k$-records which were further studied by Grudzieén and Szynal (1985), Raqab and Amin (1997) and called Type 2 k-records by Arnold et al. (1998). For $k=1$, the usual records are obtained.

Madadi and Tata (2011) presented results on the Shannon information contained in upper (lower) record values and associated record times in a sequence of independent identically distributed continuous random variables. They also considered the Shannon information contained in record data 
from an inverse sampling plan. In 2014, they generalized these results to $k$-records. Afhami and Madadi (2013) derived the exact analytical expressions for the Shannon entropy of generalized order statistics from the Pareto (IV) distribution and related distributions.

In this paper, we obtain the Shannon information contained in upper (lower) $k$-record values and $k$-record times of a random sample of size $n(n \geq k)$ and of an inverse sampling plan for Pareto-type distributions. The paper is organized as follows: In Section 2, we present some preliminary results. Section 3 contains the main results of the paper. In this section, we derive the Shannon information contained in the data consisting of all upper and lower $k$-record values and associated $k$-record times of a random sample of size $n$ and of an inverse sampling plan for Pareto-type distributions. We also present some results of the differential entropy for a finite sample of fixed size and for an inverse sampling plan. Finally, Section 4 contains a conclusion.

\section{Preliminaries}

In this section, we review some basic notations and definitions concerning Pareto-type distributions, $k$-records and entropy which will be needed in the next section.

\subsection{Pareto-type distributions}

A general version of Pareto-type distributions, called the Pareto (IV) distribution, is discussed in chapter 3 of Arnold (1983). The cumulative distribution function of this family is

$$
F_{X}(x)=1-\left[1+\left(\frac{x-\mu}{\theta}\right)^{\frac{1}{\gamma}}\right]^{-\alpha}, x>\mu,
$$

where $-\infty<\mu<\infty, \theta>0$ and $\gamma>0$ are location, scale and inequality parameters, respectively and $\alpha>0$ is the shape parameter which characterizes the tail of the distribution. This distribution is denoted by Pareto (IV) $(\mu, \theta, \gamma, \alpha)$, and its density function is as follows:

$$
f_{X}(x)=\frac{\alpha\left(\frac{x-\mu}{\theta}\right)^{\frac{1}{\gamma}-1}}{\theta \gamma\left[1+\left(\frac{x-\mu}{\theta}\right)^{\frac{1}{\gamma}}\right]^{\alpha+1}}, x>\mu .
$$

- Setting $(\alpha=1),(\gamma=1)$ and $(\gamma=1, \mu=\theta)$ in relation (2.1) and (2.2), one at a time, leads to the cumulative distribution and probability density functions of Pareto (III), Pareto (II) and Pareto (I) distributions, respectively.

- The Burr $(X I I)$ distribution is a special case of the Pareto $(I V)$ distribution in which $\mu=0, \gamma \rightarrow \frac{1}{\gamma}$. If $X \sim \operatorname{Pareto}(I V)(\mu, \theta, \gamma, \alpha)$, and $Z_{\alpha}=1+\left(\frac{x-\mu}{\theta}\right)^{\frac{1}{\gamma}}$, then

$$
F_{Z_{\alpha}}(z)=1-z^{-\alpha}, \quad z>1 \text {, }
$$

and

$$
f_{Z_{\alpha}}(z)=\frac{\alpha}{z^{\alpha+1}}, z>1,
$$

where $\alpha>0$. We denote this distribution by Pareto $(\alpha)$ and note that $Z_{\alpha}$ has the same distribution as $Z_{1}^{\frac{1}{\alpha}}$. 


\subsection{Shannon information}

Consider a discrete random variable $X$ such that $P\left\{X=x_{i}\right\}=p_{i}, i=1,2, \cdots$ and $\sum_{i=1}^{\infty} P_{i}=1$. Then

$$
H(X)=-\sum_{i=1}^{\infty} p_{i} \ln p_{i}
$$

is known as the "entropy" or Shannon information (SI) of the random variable $X$. If $X$ is a random variable having an absolutely continuous cumulative distribution function (cdf) $F(x)$ and probability density function (pdf) $f(x)$, then SI is defined as

$$
H(X)=-\int_{-\infty}^{+\infty} f(x) \ln f(x) d x .
$$

Suppose that $Z$ be a random variable with cdf $F_{Z}$, pdf $f_{Z}$ and Shannon information $H(Z)$. The following lemma is well-known and easy to check.

Lemma 2.1. If $X=\theta Z^{\gamma}+\mu$, then $F_{X}(x)=F_{Z}\left(\left(\frac{x-\mu}{\theta}\right)^{\frac{1}{\gamma}}\right)$. So,

$$
H(X)=\ln (\theta \gamma)+(\gamma-1) E(\ln Z)+H(Z), \forall \theta, \gamma>0, \mu \in \Re
$$

The Shannon entropy of the random variable $X$ is a mathematical measure of information which measures the average reduction of uncertainty of $X$. Because of its descriptive character, analytical expressions for univariate distributions have been obtained, among others, by Cover and Thomas (2006).

\section{3. $k$-record data}

Let $\left\{X_{n}: n=1,2, \ldots\right\}$ be a sequence of independent and identically distributed (i.i.d) random variables with absolutely continuous cumulative distribution function $F(x ; \theta)$ and probability density function $f(x ; \theta)$. We are interested in the Shannon information (SI) contained in the sequence of $k$-records. A $k$-record is basically the $k$-th largest observation in a partial sample. More precisely, let $X_{i: n}$ denote the $i$-th order statistic from a random sample of size $n$. We define upper $k$-record times $T_{n, k}$ and upper $k$-record values $R_{n, k}$ as follows :

(i) $T_{1, k}=k$ and $R_{1, k}=X_{1: k}$,

(ii) $T_{n, k}=\min \left\{j: j>T_{n-1, k}, X_{j}>X_{T_{n-1, k}-k+1: T_{n-1, k}}\right\}$ and $R_{n, k}=X_{T_{n, k}}-k+1: T_{n, k}, \quad(n \geq 2)$.

Suppose $N_{n, k}$ be the number of $k$-record values in $X_{1}, \ldots, X_{n}$. Lower $k$-record values, lower $k$-record times and the number of lower $k$-records are similarly defined (Arnold et al., 1998).

In the case of record and $k$-record data from a random sample of size $n$, Madadi and Tata (2011, 2014) obtained the SI contained in the last record (maximum), upper $k$-record values with associated $k$-record times, denoted by $H_{M}^{U}(n)$ and $H_{R T}^{U}(n, k)$, respectively. The SI for the lower records and $k$-records are denoted by $H_{M}^{L}(n)$ and $H_{R T}^{L}(n, k)$, respectively.

In an inverse sampling plan (ISP), one takes observations until a fixed number $m$ of $k$-records is reached. Denote the SI contained in all upper $k$-record values, the last upper $k$-record and all upper $k$-record values together with associated $k$-record times by $h_{R}^{U}(m, k), h_{M}^{U}(m, k)$ and $h_{R T}^{U}(m, k)$, respectively. The corresponding SI for lower $k$-record data are denoted by $h_{R}^{L}(m, k), h_{M}^{L}(m, k)$ and 
$h_{R T}^{L}(m, k)$, respectively.

Madadi and Tata (2011) showed that the SI contained in the last upper record is

$$
H_{M}^{U}(n)=-\ln n+\frac{n-1}{n}-n \phi_{f}^{U}(n-1,1),
$$

where

$$
\phi_{f}^{U}(i, k)=\int_{-\infty}^{+\infty}(\ln f(x))(1-F(x))^{k-1} F^{i}(x) f(x) d x .
$$

For lower record values, we have

$$
H_{M}^{L}(n)=-\ln n+\frac{n-1}{n}-n \phi_{f}^{L}(n-1,1),
$$

where $\phi_{f}^{L}(i, k)$ is obtained from $\phi_{f}^{U}(i, k)$ by replacing $F$ by $1-F$.

Madadi and Tata (2014) also proved that the SI contained in the data consisting of all upper $k$-record values and $k$-record times of a random sample of size $n(n \geq k)$ is given by

$$
\begin{aligned}
H_{R T}^{U}(n, k) & =-\left(\begin{array}{c}
n-k+2 \\
2
\end{array}\right) \ln k-k \sum_{i=0}^{n-k}\left(\begin{array}{c}
i+k-1 \\
k-1
\end{array}\right) \phi_{f}^{U}(i, k) \\
& +k \sum_{i=1}^{n-k} \sum_{j=0}^{k-1}\left(\begin{array}{c}
i+k-1 \\
k
\end{array}\right)\left(\begin{array}{c}
k-1 \\
j
\end{array}\right) \frac{(-1)^{j}}{(i+j+1)^{2}}
\end{aligned}
$$

and

$$
H_{R T}^{U}(n, 1)=\psi(n+1)+\gamma^{*}-\sum_{i=1}^{n} \frac{1}{i^{2}}-\sum_{i=0}^{n-1} \phi_{f}^{U}(i, 1),
$$

where $\gamma^{*}=0.57721566$ is the Euler constant and $\psi$ is the digamma function where

$$
\psi(x)=\frac{1}{\Gamma(x)} \int_{0}^{\infty} y^{x-1} e^{-x}(\ln y) d y .
$$

The $H_{R T}^{L}(n, k)$ and $H_{R T}^{L}(n, 1)$ contained in corresponding lower $k$-record statistics are obtained by replacing $\phi_{f}^{U}(i, k)$ and $\phi_{f}^{U}(i, 1)$ by $\phi_{f}^{L}(i, k)$ and $\phi_{f}^{L}(i, 1)$ in (2.8) and (2.9), respectively.

They also showed that the SI contained in all of $k$-record values, last upper $k$-record and all of upper $k$-record values and times of an ISP are respectively obtained as

$$
\begin{gathered}
h_{R}^{U}(m, k)=\frac{m}{k}\left(k-\frac{m+1}{2}-k \ln k\right)-\sum_{i=1}^{m} \psi_{f}^{U}(i, k), \\
h_{M}^{U}(m, k)=\ln \Gamma(m)+\frac{m(k-1)}{k}-\ln k-(m-1) \psi(m)-\psi_{f}^{U}(m, k),
\end{gathered}
$$

and for $k>1$,

$$
\begin{aligned}
h_{R T}^{U}(m, k) & =-m \ln k+\frac{m(k-1)}{k}+\left(1-\gamma^{*}-\psi(k+1)\right)\left(m+k-1-\frac{k^{m}}{(k-1)^{m-1}}\right) \\
& +\sum_{j=2}^{m-1}\left((m+k-j) k^{j-1}-\frac{k^{m}}{(k-1)^{m-j}}\right) \zeta(j, k+1)-\sum_{i=1}^{m} \psi_{f}^{U}(i, k),
\end{aligned}
$$


where

$$
\psi_{f}^{U}(i, k)=E\left(\ln f\left(R_{i, k}\right)\right)=\frac{k^{i}}{\Gamma(i)} \int_{-\infty}^{+\infty}(\ln f(x))(-\ln (1-F(x)))^{i-1}(1-F(x))^{k-1} f(x) d x .
$$

Here $\zeta(i, k)=\sum_{j=k}^{\infty} \frac{1}{j^{i}}$ is the incomplete Zeta function.

Note that for $k=1$, i.e. for ordinary records,

$$
h_{R T}^{U}(m, 1)=h_{R}^{U}(m, 1)+m(m-1)-\sum_{i=2}^{m}(m-i+1) \zeta(i) .
$$

Similar formulas hold for lower $k$-records.

\section{Shannon information in $k$-records for Pareto-type distributions}

In this section, we compute the SI in $k$-records of Pareto-type distributions for a finite sample of fixed size as well as for an inverse sampling plan.

\subsection{Shannon information in a finite sample}

Since the number of observations $n$ is fixed (and the number of records is random), the last upper record is the maximum in the sample. From (2.5) and (2.6), we have

$$
H_{M}^{U}\left(n, Z_{\alpha}\right)=-\ln n+\frac{n-1}{n}+\frac{\alpha+1}{\alpha}\left(\psi(n+1)+\gamma^{*}\right)-\ln \alpha,
$$

and

$$
H_{M}^{L}\left(n, Z_{\alpha}\right)=-\ln n+\frac{n-1}{n}+\frac{\alpha+1}{n \alpha}-\ln \alpha .
$$

Therefore the SI contained in the last record for the Pareto $(I V)$ are

$$
\begin{aligned}
H_{M}^{U}(n, X) & =H_{M}^{U}\left(n, Z_{\alpha}\right)+\ln (\theta \gamma)-\frac{1-\gamma}{\alpha}\left(\psi(n+1)+\gamma^{*}\right) \\
& +n \alpha(1-\gamma) \sum_{j=0}^{n-1} \sum_{l=1}^{\infty}\left(\begin{array}{c}
n-1 \\
j
\end{array}\right) \frac{(-1)^{j}}{l(l+j \alpha+\alpha)},
\end{aligned}
$$

and

$$
H_{M}^{L}(n, X)=H_{M}^{L}\left(n, Z_{\alpha}\right)+\ln (\theta \gamma)-n(1-\gamma)\left[\frac{1}{n^{2} \alpha}-\alpha \sum_{l=1}^{\infty} \frac{1}{l(l+n \alpha)}\right] .
$$

When $\alpha$ is integer,

$$
\sum_{l=1}^{\infty} \frac{1}{l(l+j \alpha+\alpha)}=\frac{1}{j \alpha+\alpha}\left(\psi(j \alpha+\alpha+1)+\gamma^{*}\right)
$$

and

$$
\sum_{l=1}^{\infty} \frac{1}{l(l+n \alpha)}=\frac{1}{n \alpha}\left(\psi(n \alpha+1)+\gamma^{*}\right) .
$$

Let 


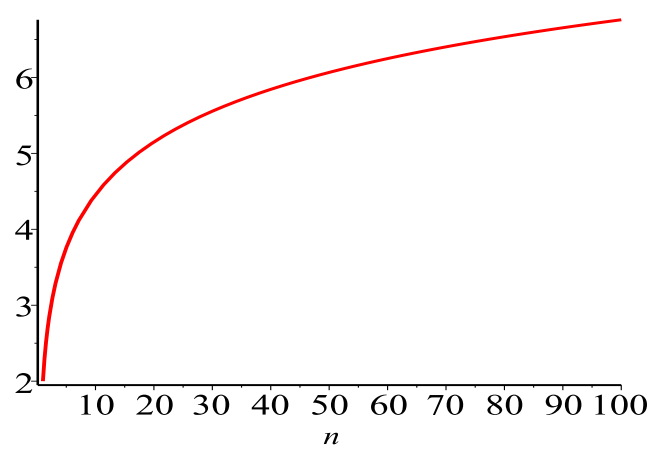

(a)

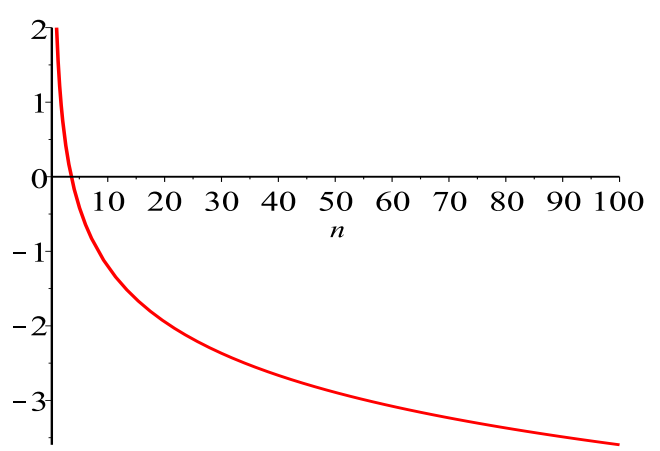

(b)

Fig. 1. (a) and (b) represent $H_{M}^{U}\left(n, Z_{1}\right)$ and $H_{M}^{L}\left(n, Z_{1}\right)$, respectively.

$$
\Delta H_{M}^{U}\left(n, Z_{\alpha}\right)=H_{M}^{U}\left(n+1, Z_{\alpha}\right)-H_{M}^{U}\left(n, Z_{\alpha}\right)
$$

and

$$
\Delta H_{M}^{L}\left(n, Z_{\alpha}\right)=H_{M}^{L}\left(n+1, Z_{\alpha}\right)-H_{M}^{L}\left(n, Z_{\alpha}\right),
$$

denote the $n$-th differential of the entropy, that is, the change in the entropy when a new observation occurs. From (3.1) and (3.2), we obtain

$$
\Delta H_{M}^{U}\left(n, Z_{\alpha}\right)=\ln \frac{n}{n+1}+\frac{1}{n}+\frac{1}{(n+1) \alpha},
$$

and

$$
\Delta H_{M}^{L}\left(n, Z_{\alpha}\right)=\ln \frac{n}{n+1}-\frac{1}{n(n+1) \alpha} .
$$

Fig. 1. represent behaviours of $H_{M}^{U}\left(n, Z_{1}\right)$ and $H_{M}^{L}\left(n, Z_{1}\right)$ with respect to $n$.

One can easily see that

(i) $\lim _{n \rightarrow \infty} \Delta H_{M}^{U}\left(n, Z_{\alpha}\right)=\lim _{n \rightarrow \infty} \Delta H_{M}^{L}\left(n, Z_{\alpha}\right)=0$.

(ii) $\Delta H_{M}^{U}\left(n, Z_{\alpha}\right)$ and $\Delta H_{M}^{L}\left(n, Z_{\alpha}\right)$ are respectively always nonnegative and negative functions i.e. $H_{M}^{U}\left(n, Z_{\alpha}\right)$ and $H_{M}^{L}\left(n, Z_{\alpha}\right)$ are increasing and decreasing functions with respect to sample size for each $\alpha$. Also, $\Delta H_{M}^{U}\left(n, Z_{\alpha}\right)$ and $\Delta H_{M}^{L}\left(n, Z_{\alpha}\right)$ are decreasing and increasing functions of $n$ for each $\alpha$.

(iii) $0 \leq \Delta H_{M}^{U}\left(n, Z_{\alpha}\right)<-\ln 2+1+\frac{1}{2 \alpha}$ and $-\ln 2-\frac{1}{2 \alpha}<\Delta H_{M}^{L}\left(n, Z_{\alpha}\right) \leq 0$.

(iv) For each fixed $n, \Delta H_{M}^{U}\left(n, Z_{\alpha}\right)$ and $\Delta H_{M}^{L}\left(n, Z_{\alpha}\right)$ are decreasing convex and increasing concave functions of $\alpha$, respectively.

(v) $H_{M}^{U}\left(n, Z_{\alpha}\right)$ and $H_{M}^{L}\left(n, Z_{\alpha}\right)$ are decreasing convex functions of $\alpha$ for each $n$.

Theorem 3.1. For Pareto (1), the SI contained in all the upper and lower k-record values together with $k$-record times of a random sample of size $n(n \geq k)$ are equal to

$$
H_{R T}^{U}\left(n, k, Z_{1}\right)=-\left(\begin{array}{c}
n-k+2 \\
2
\end{array}\right) \ln k+\frac{2}{k}+\sum_{i=1}^{n-k}\left(\begin{array}{c}
i+k-1 \\
k-1
\end{array}\right)(2 k I(k: k+i)+i I(i+1: k+i)),
$$


and

$$
H_{R T}^{L}\left(n, k, Z_{1}\right)=-\left(\begin{array}{c}
n-k+2 \\
2
\end{array}\right) \ln k+2 k I(1: k)+\sum_{i=1}^{n-k}\left(\begin{array}{c}
i+k-1 \\
k-1
\end{array}\right)(2 k+i) I(i+1: k+i)
$$

where $I(j: n)=\sum_{i=0}^{n-j}\left(\begin{array}{c}n-j \\ i\end{array}\right) \frac{(-1)^{i}}{(i+j)^{2}}, 0<j \leq n$.

Also for $k=1$, we obtain

$$
H_{R T}^{U}\left(n, 1, Z_{1}\right)=\left(1+2 \gamma^{*}\right)\left(\psi(n+1)+\gamma^{*}\right)-\sum_{i=1}^{n} \frac{1}{i^{2}}+2 \sum_{i=1}^{n} \frac{\psi(i+1)}{i}
$$

and

$$
H_{R T}^{L}\left(n, 1, Z_{1}\right)=\left(\psi(n+1)+\gamma^{*}\right)+\sum_{i=1}^{n} \frac{1}{i^{2}}
$$

From (2.3), (2.4) and (2.6), we get

$$
\phi_{f}^{U}\left(i, k, Z_{1}\right)=\int_{0}^{1}(2 \ln u) u^{k-1}(1-u)^{i} d u=2 \sum_{j=0}^{i}\left(\begin{array}{l}
i \\
j
\end{array}\right) \frac{(-1)^{j}}{(j+k)^{2}}=-2 I(k: k+i) .
$$

Substituting this expression in (2.8), we obtain

$$
\begin{aligned}
H_{R T}^{U}\left(n, k, Z_{1}\right)= & -\left(\begin{array}{c}
n-k+2 \\
2
\end{array}\right) \ln k+2 k \sum_{i=0}^{n-k}\left(\begin{array}{c}
i+k-1 \\
k-1
\end{array}\right) I(k: k+i)+k \sum_{i=1}^{n-k}\left(\begin{array}{c}
i+k-1 \\
k
\end{array}\right) \\
& I(i+1: k+i)=-\left(\begin{array}{c}
n-k+2 \\
2
\end{array}\right) \ln k+2 k \cdot \frac{1}{k^{2}}+k \sum_{i=1}^{n-k}\left[2\left(\begin{array}{c}
i+k-1 \\
k-1
\end{array}\right)\right. \\
& \left.I(k: k+i)+\left(\begin{array}{c}
i+k-1 \\
k
\end{array}\right) I(i+1: k+i)\right],
\end{aligned}
$$

hence, we conclude (3.3). Similarly,

$$
\phi_{f}^{L}\left(i, k, Z_{1}\right)=-2 I(i+1: k+i),
$$

therefore (3.4) is obtained. From (2.6), and recalling that $-\ln (1-t)=\sum_{q=1}^{\infty} \frac{t^{q}}{q}$, we have

$$
\phi_{f}^{U}\left(i, 1, Z_{1}\right)=-\frac{2\left(\psi(i+2)+\gamma^{*}\right)}{i+1}
$$

and

$$
\phi_{f}^{L}\left(i, 1, Z_{1}\right)=-\frac{2}{(i+1)^{2}}
$$

thus (3.5) and (3.6) are achieved. 
Corollary 3.1. For $Z_{\alpha}=Z_{1}^{\frac{1}{\alpha}}$, we obtain

$$
\begin{aligned}
H_{R T}^{U}\left(n, k, Z_{\alpha}\right) & =H_{R T}^{U}\left(n, k, Z_{1}\right)-k(\ln \alpha)(\psi(n+1)-\psi(k)) \\
& +k\left(\frac{1}{\alpha}-1\right) \sum_{i=0}^{n-k}\left(\begin{array}{c}
i+k-1 \\
k-1
\end{array}\right) I(k: k+i),
\end{aligned}
$$

and

$$
\begin{aligned}
H_{R T}^{L}\left(n, k, Z_{\alpha}\right) & =H_{R T}^{L}\left(n, k, Z_{1}\right)-k(\ln \alpha)(\psi(n+1)-\psi(k)) \\
& +k\left(\frac{1}{\alpha}-1\right) \sum_{i=0}^{n-k}\left(\begin{array}{c}
i+k-1 \\
k-1
\end{array}\right) I(i+1: k+i) .
\end{aligned}
$$

Also,

$$
H_{R T}^{U}\left(n, 1, Z_{\alpha}\right)=H_{R T}^{U}\left(n, 1, Z_{1}\right)-(\ln \alpha)\left(\psi(n+1)+\gamma^{*}\right)+\left(\frac{1}{\alpha}-1\right) \sum_{i=0}^{n-1} I(1: i+1),
$$

and

$$
H_{R T}^{L}\left(n, 1, Z_{\alpha}\right)=H_{R T}^{L}\left(n, 1, Z_{1}\right)-(\ln \alpha)\left(\psi(n+1)+\gamma^{\star}\right)+\left(\frac{1}{\alpha}-1\right) \sum_{i=1}^{n} \frac{1}{i^{2}} .
$$

Corollary 3.2. The SI contained in all k-record values and k-record times of a random sample of size $n(n \geq k)$ from the Pareto $(I V)$ distribution are

$$
\begin{array}{r}
H_{R T}^{U}(n, k, X)=H_{R T}^{U}\left(n, k, Z_{\alpha}\right)+k(\ln (\theta \gamma))(\psi(n+1)-\psi(k))-\frac{k(1-\gamma)}{\alpha} \sum_{i=0}^{n-k}\left(\begin{array}{c}
i+k-1 \\
k-1
\end{array}\right) \\
I(k: i+k)+k \alpha(1-\gamma) \sum_{i=0}^{n-k} \sum_{j=0}^{i} \sum_{q=1}^{\infty}\left(\begin{array}{c}
i+k-1 \\
k-1
\end{array}\right)\left(\begin{array}{c}
i \\
j
\end{array}\right) \frac{(-1)^{j}}{q(q+k \alpha+j \alpha)},
\end{array}
$$

and

$$
\begin{aligned}
H_{R T}^{L}(n, k, X)= & H_{R T}^{L}\left(n, k, Z_{\alpha}\right)+k(\ln (\theta \gamma))(\psi(n+1)-\psi(k))+k \alpha(1-\gamma) \sum_{i=0}^{n-k} \sum_{j=0}^{k-1} \sum_{q=1}^{\infty}\left(\begin{array}{c}
i+k-1 \\
k-1
\end{array}\right) \\
& \left(\begin{array}{c}
k-1 \\
j
\end{array}\right) \frac{(-1)^{j}}{q(q+i \alpha+j \alpha+\alpha)}-\frac{k(1-\gamma)}{\alpha} \sum_{i=0}^{n-k} \sum_{j=0}^{k-1}\left(\begin{array}{c}
i+k-1 \\
k-1
\end{array}\right)\left(\begin{array}{c}
k-1 \\
j
\end{array}\right) \frac{(-1)^{j}}{(i+j+k)^{2}},
\end{aligned}
$$

respectively.

For ordinary records, these information can be written as

$$
\begin{aligned}
H_{R T}^{U}(n, 1, X)= & H_{R T}^{U}\left(n, 1, Z_{\alpha}\right)+\left(\ln (\theta \gamma)-\frac{\gamma^{*}(1-\gamma)}{\alpha}\right)\left(\psi(n+1)+\gamma^{\star}\right)-\frac{1-\gamma}{\alpha} \\
& \sum_{i=1}^{n} \frac{\psi(i+1)}{i}+\alpha(1-\gamma) \sum_{i=0}^{n-1} \sum_{j=0}^{i} \sum_{q=1}^{\infty}\left(\begin{array}{l}
i \\
j
\end{array}\right) \frac{(-1)^{j}}{q(q+j \alpha+\alpha)},
\end{aligned}
$$

and 

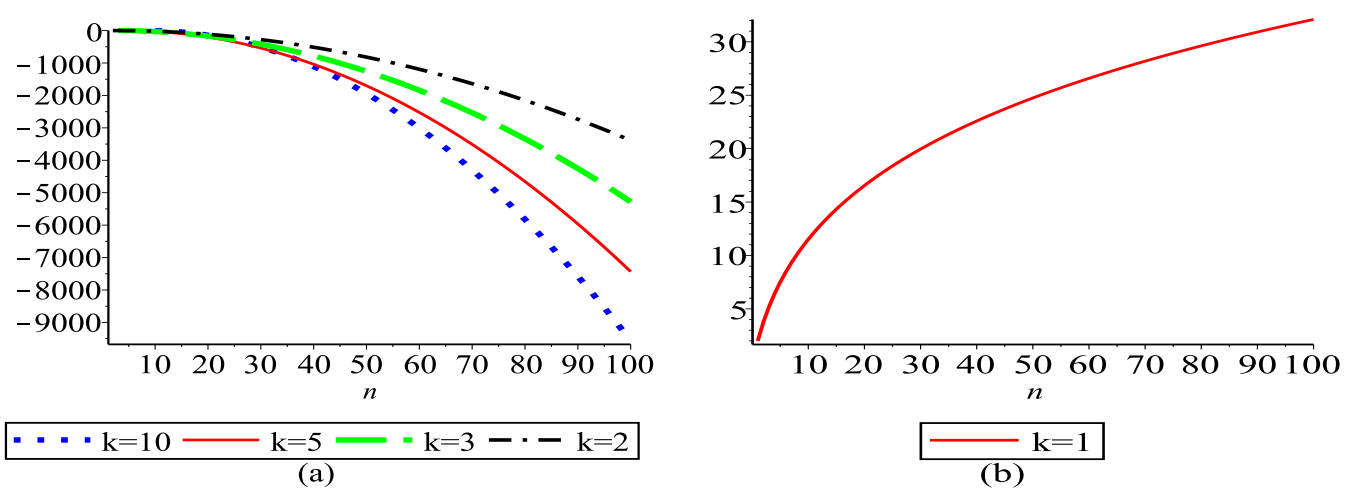

Fig. 2. (a) and (b) represent $H_{R T}^{U}\left(n, k, Z_{1}\right)$ and $H_{R T}^{U}\left(n, 1, Z_{1}\right)$, respectively.

$$
\begin{aligned}
H_{R T}^{L}(n, 1, X) & =H_{R T}^{L}\left(n, 1, Z_{\alpha}\right)+(\ln (\theta \gamma))\left(\psi(n+1)+\gamma^{*}\right) \\
& -\frac{1-\gamma}{\alpha} \sum_{i=1}^{n} \frac{1}{i^{2}}+\alpha(1-\gamma) \sum_{i=0}^{n-1} \sum_{q=1}^{\infty} \frac{1}{q(q+i \alpha+\alpha)}
\end{aligned}
$$

When $\alpha$ is integer, we have

$$
\sum_{q=1}^{\infty} \frac{1}{q(q+i \alpha+\alpha)}=\frac{1}{i \alpha+\alpha}\left(\psi(i \alpha+\alpha+1)+\gamma^{*}\right)
$$

For $n=k, k+1, \cdots$, let

$$
\Delta H_{R T}^{U}(n, k)=H_{R T}^{U}(n+1, k)-H_{R T}^{U}(n, k),
$$

and

$$
\Delta H_{R T}^{L}(n, k)=H_{R T}^{L}(n+1, k)-H_{R T}^{L}(n, k),
$$

denote the $n$-th SI differential corresponding to entropies in equations (3.3) and (3.4), then it is easy to see that

$$
\Delta H_{R T}^{U}\left(n, k, Z_{1}\right)=-(n-k+2) \ln k+\left(\begin{array}{c}
n \\
k-1
\end{array}\right)[2 k I(k: n+1)+(n-k+1) I(n-k+2: n+1)],
$$

and

$$
\Delta H_{R T}^{L}\left(n, k, Z_{1}\right)=-(n-k+2) \ln k+\left(\begin{array}{c}
n \\
k-1
\end{array}\right)(n+k+1) I(n-k+2: n+1) .
$$

Fig. 2. displays $H_{R T}^{U}\left(n, k, Z_{1}\right)$ and $H_{R T}^{U}\left(n, 1, Z_{1}\right)$ for several values of $k$ and $n$. Behaviours of $H_{R T}^{L}\left(n, k, Z_{1}\right)$ and $H_{R T}^{L}\left(n, 1, Z_{1}\right)$ are shown in Fig. 3. Our numerical computations suggest that when 


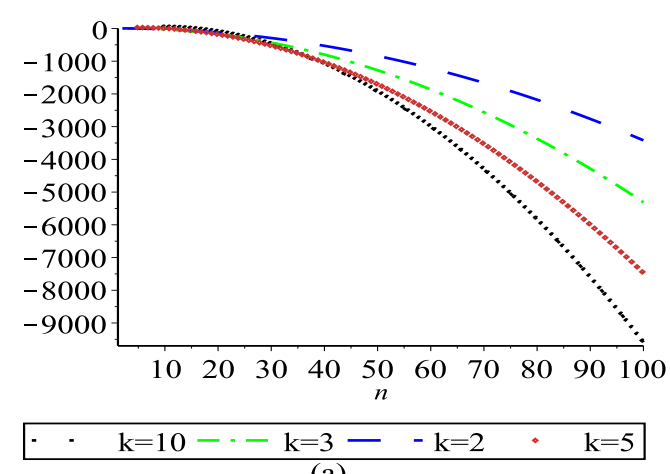

(a)

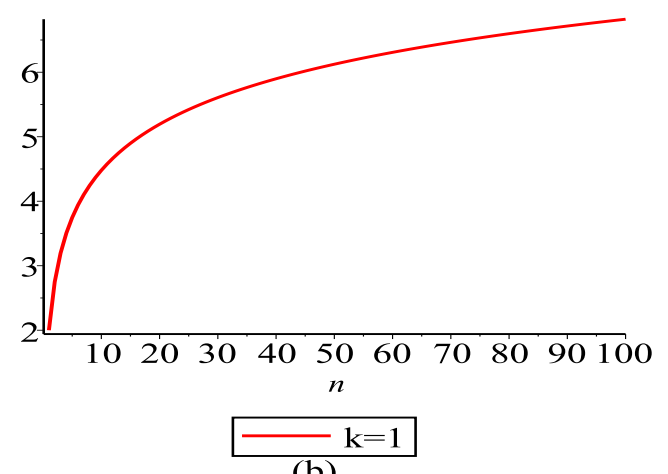

(b)

Fig. 3. (a) and (b) represent $H_{R T}^{L}\left(n, k, Z_{1}\right)$ and $H_{R T}^{L}\left(n, 1, Z_{1}\right)$, respectively.

$k \geq 2$ is fixed, the differential entropy is negative and decreasing in $n$. Also, for $k=1$, we have

$$
\Delta H_{R T}^{U}\left(n, 1, Z_{1}\right)=\frac{n+2}{(n+1)^{2}}+\frac{2}{n+1}\left(\psi(n+1)+\gamma^{*}\right),
$$

and

$$
\Delta H_{R T}^{L}\left(n, 1, Z_{1}\right)=\frac{n+2}{(n+1)^{2}} .
$$

The following properties are easy to check and confirm via Figs. 2. and 3.

(i) $\lim _{n \rightarrow \infty} \Delta H_{R T}^{U}\left(n, 1, Z_{1}\right)=\lim _{n \rightarrow \infty} \Delta H_{R T}^{L}\left(n, 1, Z_{1}\right)=0$.

(ii) $\Delta H_{R T}^{U}\left(n, 1, Z_{1}\right)$ and $\Delta H_{R T}^{L}\left(n, 1, Z_{1}\right)$ are non-negative decreasing functions of $n$.

(iii) $0 \leq \Delta H_{R T}^{L}\left(n, 1, Z_{1}\right)<\frac{3}{4}$.

We note that $\psi(n+1)=\psi(n)+\frac{1}{n}$ and $\lim _{n \rightarrow \infty} \frac{\psi(n)}{n}=0$.

\subsection{Shannon information in an inverse sampling plan}

In an inverse sampling plan, we take observations until a fixed number $m$ of $k$-records is reached. Here, we compute the SI of $k$-records for an inverse sampling plan from Pareto-type distributions and discuss their properties.

Theorem 3.2. The SI contained in the $m$-th upper and $m$-th lower $k$-record values from $Z_{1}$ are given by

$$
h_{M}^{U}\left(m, k, Z_{1}\right)=\ln \Gamma(m)+\frac{m(k+1)}{k}-\ln k-(m-1) \psi(m), m \geq 1,
$$

and

$$
\begin{aligned}
h_{M}^{L}\left(m, k, Z_{1}\right) & =\ln \Gamma(m)+\frac{m(k-1)}{k}-\ln k-(m-1) \psi(m) \\
& +2\left(\psi(k+1)+\gamma^{*}-\sum_{j=2}^{m} k^{j-1} \zeta(j, k+1)\right), m \geq 2 .
\end{aligned}
$$


It is easy to check (3.7). Now from (2.13), replacing $F$ by $1-F$, we obtain

$$
\psi_{f}^{L}\left(i, k, Z_{1}\right)=-2 \frac{k^{i}}{\Gamma(i)} \int_{0}^{1}(-\ln (1-y))(-\ln y)^{i-1} y^{k-1} d y
$$

Since $-\ln (1-y)=\sum_{l=1}^{\infty} \frac{y^{l}}{l}$, the above expression can be written as

$$
\begin{aligned}
\psi_{f}^{L}\left(i, k, Z_{1}\right) & =-2 k^{i} \sum_{l=k+1}^{\infty} \frac{1}{(l-k) l^{i}}=-2 k^{i}\left[\frac{1}{k^{i}} \sum_{j=1}^{k} \frac{1}{j}-\frac{\zeta(2, k+1)}{k^{i-1}}-\cdots-\frac{\zeta(i, k+1)}{k}\right] \\
& =-2\left[\psi(k+1)+\gamma^{*}-\sum_{j=2}^{i} k^{j-1} \zeta(j, k+1)\right] .
\end{aligned}
$$

It is clear that $i=1$, leads to

$$
\psi_{f}^{L}\left(1, k, Z_{1}\right)=-2 k \sum_{j=k+1}^{\infty} \frac{1}{j(j-k)}=-2\left[\sum_{j=1}^{\infty} \frac{1}{j}-\sum_{j=k+1}^{\infty} \frac{1}{j}\right]=-2 \sum_{j=1}^{k} \frac{1}{j}=-2\left(\psi(k+1)+\gamma^{*}\right) .
$$

Substituting (3.9) and (3.10) in (2.11), the proof is complete.

Remark 3.1. For $m=1$, we have

$$
h_{M}^{L}\left(1, k, Z_{1}\right)=h_{R}^{L}\left(1, k, Z_{1}\right)=h_{R T}^{L}\left(1, k, Z_{1}\right)=\frac{k-1}{k}-\ln k+2\left(\psi(k+1)+\gamma^{*}\right) .
$$

Corollary 3.3. For $Z_{\alpha}$, we obtain

$$
h_{M}^{U}\left(m, k, Z_{\alpha}\right)=h_{M}^{U}\left(m, k, Z_{1}\right)-\ln \alpha+\left(\frac{1}{\alpha}-1\right) \frac{m}{k},
$$

and

$$
h_{M}^{L}\left(m, k, Z_{\alpha}\right)=h_{M}^{L}\left(m, k, Z_{1}\right)-\ln \alpha+\left(\frac{1}{\alpha}-1\right)\left(\psi(k+1)+\gamma^{*}-\sum_{j=2}^{m} k^{j-1} \zeta(j, k+1)\right) .
$$

Also, $h_{M}^{L}\left(1, k, Z_{\alpha}\right)=h_{M}^{L}\left(1, k, Z_{1}\right)-\ln \alpha+\left(\frac{1}{\alpha}-1\right)\left(\psi(k+1)+\gamma^{*}\right)$.

Corollary 3.4. The SI contained in the $m$-th upper and $m$-th lower $k$-record values from the Pareto (IV) are

$$
h_{M}^{U}(m, k, X)=h_{M}^{U}\left(m, k, Z_{\alpha}\right)+\ln (\theta \gamma)+(k \alpha)^{m}(1-\gamma) \sum_{q=k \alpha+1}^{\infty} \frac{1}{(q-k \alpha) q^{m}}-(1-\gamma) \frac{m}{k \alpha}
$$

and

$$
\begin{aligned}
h_{M}^{L}(m, k, X)= & h_{M}^{L}\left(m, k, Z_{\alpha}\right)+\ln (\theta \gamma)+\frac{1-\gamma}{\alpha}\left(\psi(k+1)+\gamma^{*}\right)-\frac{(1-\gamma)}{\alpha} \\
& \sum_{j=2}^{m} k^{j-1} \zeta(j, k+1)+(1-\gamma) k^{m} \sum_{j=1}^{\infty} \sum_{q=0}^{\infty}\left(\begin{array}{c}
\frac{j}{\alpha} \\
q
\end{array}\right) \frac{(-1)^{q}}{j(q+k)^{m}},
\end{aligned}
$$


respectively. When $\alpha$ is integer, one obtains

$$
\begin{aligned}
h_{M}^{U}(m, k, X)= & h_{M}^{U}\left(m, k, Z_{\alpha}\right)+\ln (\theta \gamma)+(1-\gamma)\left(\psi(k \alpha+1)+\gamma^{*}\right) \\
& -(1-\gamma) \sum_{j=2}^{m}(k \alpha)^{j-1} \zeta(j, k \alpha+1)-(1-\gamma) \frac{m}{k \alpha} .
\end{aligned}
$$

Let

$$
\Delta h_{M}^{U}(m, k)=h_{M}^{U}(m+1, k)-h_{M}^{U}(m, k),
$$

and

$$
\Delta h_{M}^{L}(m, k)=h_{M}^{L}(m+1, k)-h_{M}^{L}(m, k),
$$

denote the change in entropy in the last upper and last lower $k$-record values, respectively when the new $k$-record is observed. Then, from (3.7) and (3.8), we obtain

$$
\Delta h_{M}^{U}\left(m, k, Z_{1}\right)=\ln m-\psi(m)+\frac{1}{k},
$$

and

$$
\Delta h_{M}^{L}\left(m, k, Z_{1}\right)=\ln m-\psi(m)-\frac{1}{k}-2 k^{m} \zeta(m+1, k+1) .
$$

It is easy to see that

(i) $\Delta h_{M}^{U}\left(m, k, Z_{1}\right)$ is a positive function of $m$ for each fixed $k$. Hence the sequence of functions $\left\{h_{M}^{U}\left(m, k, Z_{1}\right)\right\}$ is increasing with respect to $m$. Also, $\Delta h_{M}^{L}\left(m, k, Z_{1}\right)$ is a negative function of $m$, for each fixed $k$. Therefore $\left\{h_{M}^{L}\left(m, k, Z_{1}\right)\right\}$ is a sequence of decreasing functions of $m$.

(ii) for each fixed $k, \Delta h_{M}^{U}\left(m, k, Z_{1}\right)$ and $\Delta h_{M}^{L}\left(m, k, Z_{1}\right)$ are decreasing and increasing functions of $m$, respectively.

(iii) $\lim _{m \rightarrow \infty} \Delta h_{M}^{U}\left(m, k, Z_{1}\right)=\frac{1}{k}$ and $\lim _{m \rightarrow \infty} \Delta h_{M}^{L}\left(m, k, Z_{1}\right)=-\frac{1}{k}$.

(iv) $\frac{1}{2 m}+\frac{1}{k}<\Delta h_{M}^{U}\left(m, k, Z_{1}\right)<\frac{1}{m}+\frac{1}{k}$ and $-\frac{1}{k}-2 k \zeta(2, k+1)+\gamma^{*}<\Delta h_{M}^{L}\left(m, k, Z_{1}\right)<-\frac{1}{k}$, for each $m \geq 2$.

We notice that $\frac{1}{2 m}<\ln m-\psi(m)<\frac{1}{m}$, for $m>0, \psi(1)=-\gamma^{*}$ and $\lim _{m \rightarrow \infty} k^{m} \zeta(m+1, k+1)=0$. For $k=1$, we obtain

$$
\Delta h_{M}^{U}\left(m, 1, Z_{1}\right)=\ln m-\psi(m)+1,
$$

and

$$
\Delta h_{M}^{L}\left(m, 1, Z_{1}\right)=\ln m-\psi(m)-2 \zeta(m+1)+1 .
$$

It is easy to prove that

(i) $\lim _{m \rightarrow \infty} \Delta h_{M}^{U}\left(m, 1, Z_{1}\right)=1$ and $\lim _{m \rightarrow \infty} \Delta h_{M}^{L}\left(m, 1, Z_{1}\right)=-1$.

(ii) $\frac{1}{2 m}+1<\Delta h_{M}^{U}\left(m, 1, Z_{1}\right)<\frac{1}{m}+1$, for each $m \geq 2$ and $\Delta h_{M}^{L}\left(m, 1, Z_{1}\right)>\frac{1}{2 m}-\frac{\pi^{2}}{3}+1$.

(iii) $\Delta h_{M}^{U}\left(m, 1, Z_{1}\right)$ is a positive decreasing function of $m$, therefore $h_{M}^{U}\left(m, 1, Z_{1}\right)$ is an increasing function of $m$. 

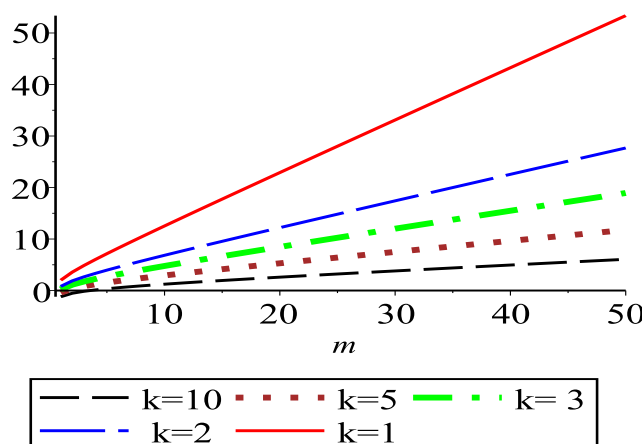

(a)
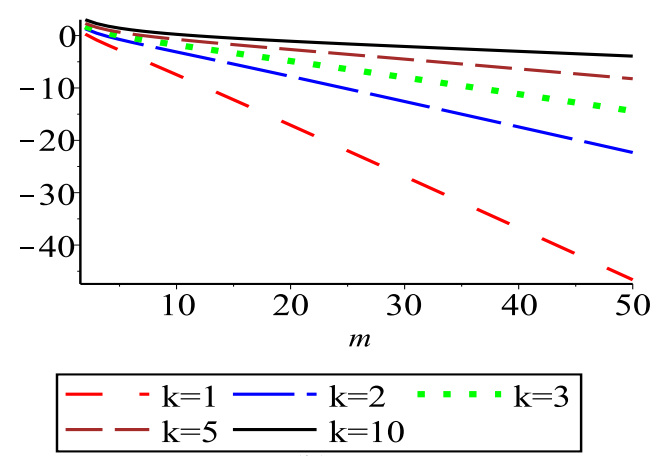

(b)

Fig. 4. (a) and (b) represent $h_{M}^{U}\left(m, k, Z_{1}\right)$ and $h_{M}^{L}\left(m, k, Z_{1}\right)$, respectively.

Fig. 4. displays a graphical representation of $h_{M}^{U}\left(m, k, Z_{1}\right)$ and $h_{M}^{L}\left(m, k, Z_{1}\right)$ when $k=1,2,3,5,10$. Let

$$
\Delta^{*} h_{M}^{U}(m, k)=h_{M}^{U}(m, k+1)-h_{M}^{U}(m, k)
$$

and

$$
\Delta^{*} h_{M}^{L}(m, k)=h_{M}^{L}(m, k+1)-h_{M}^{L}(m, k),
$$

denote the SI difference of $(k+1)$-record and $k$-record in the $m$-th upper and $m$-th lower cases, respectively. Then, from (3.7) and (3.8), it is easy to check

$$
\Delta^{*} h_{M}^{U}\left(m, k, Z_{1}\right)=\ln \frac{k}{k+1}-\frac{m}{k(k+1)},
$$

and

$$
\Delta^{*} h_{M}^{L}\left(m, k, Z_{1}\right)=\frac{m+2 k}{k(k+1)}+\ln \frac{k}{k+1}+\frac{2}{k} \sum_{j=2}^{m}\left(\frac{k}{k+1}\right)^{j}+2 \sum_{j=2}^{m}\left(k^{j-1}-(k+1)^{j-1}\right) \zeta(j, k+2) .
$$

We note that $\zeta(j, k+1)=\frac{1}{(k+1)^{j}}+\zeta(j, k+2)$. Hence

(i) $\Delta^{*} h_{M}^{U}\left(m, k, Z_{1}\right)$ is a negative increasing function of $k$, for each fixed $m$. Hence, $h_{M}^{U}\left(m, k, Z_{1}\right)$ is a monotone decreasing function of $k$. Similarly, $\Delta^{*} h_{M}^{L}\left(m, k, Z_{1}\right)$ is a positive decreasing function of $k$ and therefore $h_{M}^{L}\left(m, k, Z_{1}\right)$ is a monotone increasing function of $k$ for each $m$.

(ii) $\lim _{k \rightarrow \infty} \Delta^{*} h_{M}^{U}\left(m, k, Z_{1}\right)=\lim _{k \rightarrow \infty} \Delta^{*} h_{M}^{L}\left(m, k, Z_{1}\right)=0$.

(iii) $-\ln 2-\frac{m}{2}<\Delta^{*} h_{M}^{U}\left(m, k, Z_{1}\right)<0$ and $0<\Delta^{*} h_{M}^{L}\left(m, k, Z_{1}\right)<\frac{m}{2}+2-\ln 2-\left(\frac{1}{2}\right)^{m-1}+\sum_{j=2}^{m}(2-$ $\left.2^{j}\right) \zeta(j, 3)$.

Remark 3.2. For $m=1$, we have

$$
\Delta^{*} h_{M}^{L}\left(1, k, Z_{1}\right)=\ln \frac{k}{k+1}+\frac{2 k+1}{k(k+1)} .
$$

Therefore

(i) $\lim _{k \rightarrow \infty} \Delta^{*} h_{M}^{L}\left(1, k, Z_{1}\right)=0$ and $0<\Delta^{*} h_{M}^{L}\left(1, k, Z_{1}\right)<-\ln 2+\frac{3}{2}$. 
(ii) $\Delta^{*} h_{M}^{L}\left(1, k, Z_{1}\right)$ is positive monotone decreasing function of $k$ for each $m$. So $h_{M}^{L}\left(1, k, Z_{1}\right)$ is a monotone increasing function of $k$.

Theorem 3.3. The SI contained in all of the first $m$ upper and $m$ lower $k$-record values of an ISP are respectively given by

$$
h_{R}^{U}\left(m, k, Z_{1}\right)=\frac{m}{k}\left(k+\frac{m+1}{2}-k \ln k\right)
$$

and

$$
h_{R}^{L}\left(m, k, Z_{1}\right)=\frac{m}{k}\left(k-\frac{m+1}{2}-k \ln k\right)+2 m\left(\psi(k+1)+\gamma^{*}\right)-2 \sum_{j=2}^{m}(m-j+1) k^{j-1} \zeta(j, k+1) .
$$

Corollary 3.5. For Pareto (I) with parameter $\alpha$, we have

$$
h_{R}^{U}\left(m, k, Z_{\alpha}\right)=h_{R}^{U}\left(m, k, Z_{1}\right)-m \ln \alpha+\left(\frac{1}{\alpha}-1\right) \frac{m(m+1)}{2 k},
$$

and

$$
\begin{aligned}
h_{R}^{L}\left(m, k, Z_{\alpha}\right) & =h_{R}^{L}\left(m, k, Z_{1}\right)-m \ln \alpha+m\left(\frac{1}{\alpha}-1\right)\left(\psi(k+1)+\gamma^{*}\right) \\
& +\left(1-\frac{1}{\alpha}\right) \sum_{j=2}^{m}(m-j+1) k^{j-1} \zeta(j, k+1) .
\end{aligned}
$$

Corollary 3.6. For the Pareto (IV), one can obtains

$$
h_{R}^{U}(m, k, X)=h_{R}^{U}\left(m, k, Z_{\alpha}\right)+m \ln (\theta \gamma)+(1-\gamma) \sum_{j=1}^{m} \sum_{q=k \alpha+1}^{\infty} \frac{(k \alpha)^{j}}{(q-k \alpha) q^{j}}-\frac{m(m+1)(1-\gamma)}{2 k \alpha},
$$

and

$$
\begin{aligned}
h_{R}^{L}(m, k, X)= & h_{R}^{L}\left(m, k, Z_{\alpha}\right)+m \ln (\theta \gamma)+\frac{m(1-\gamma)}{\alpha}\left(\psi(k+1)+\gamma^{*}\right)-\frac{1-\gamma}{\alpha} \sum_{j=2}^{m}(m-j+1) \\
& k^{j-1} \zeta(j, k+1)+(1-\gamma) \sum_{j=1}^{m} \sum_{l=1}^{\infty} \sum_{q=0}^{\infty}\left(\begin{array}{c}
\frac{l}{\alpha} \\
q
\end{array}\right) \frac{(-1)^{q} k^{j}}{l(q+k)^{j}} .
\end{aligned}
$$

When $\alpha$ is integer,

$$
\begin{aligned}
h_{R}^{U}(m, k, X) & =h_{R}^{U}\left(m, k, Z_{\alpha}\right)+m \ln (\theta \gamma)+m(1-\gamma)\left(\psi(k \alpha+1)+\gamma^{*}\right) \\
& -(1-\gamma) \sum_{j=2}^{m}(m-j+1)(k \alpha)^{j-1} \zeta(j, k \alpha+1)-\frac{m(m+1)(1-\gamma)}{2 k \alpha} .
\end{aligned}
$$

Let

$$
\Delta h_{R}^{U}(m, k)=h_{R}^{U}(m+1, k)-h_{R}^{U}(m, k),
$$

and

$$
\Delta h_{R}^{L}(m, k)=h_{R}^{L}(m+1, k)-h_{R}^{L}(m, k),
$$

denotes the change in entropy in observing the $k$-record values from the $m$-th to the $(m+1)$-th $k$-record values. Therefore, 


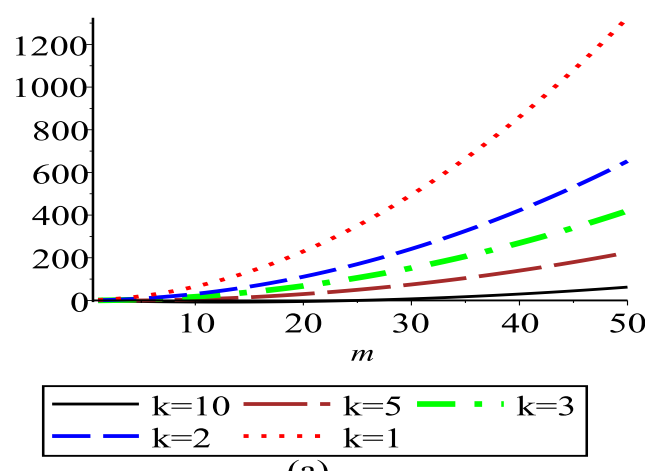

(a)

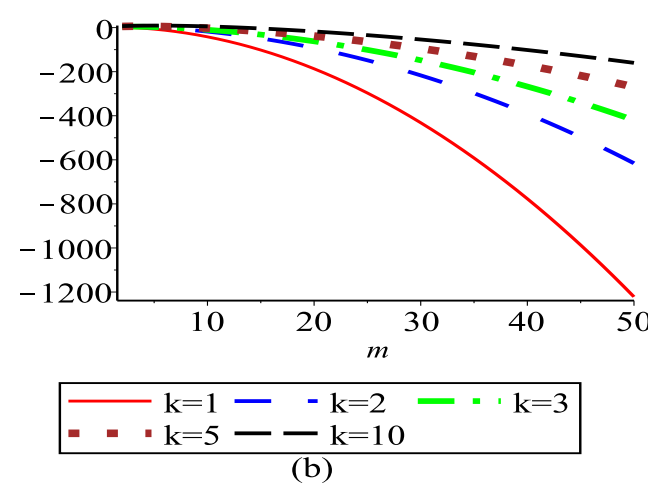

(b)

Fig. 5. (a) and (b) represent $h_{R}^{U}\left(m, k, Z_{1}\right)$ and $h_{R}^{L}\left(m, k, Z_{1}\right)$, respectively.

$$
\Delta h_{R}^{U}\left(m, k, Z_{1}\right)=\frac{m+1}{k}-\ln k+1
$$

and

$$
\Delta h_{R}^{L}\left(m, k, Z_{1}\right)=2\left(\psi(k+1)+\gamma^{*}\right)-2 \sum_{j=2}^{m+1} k^{j-1} \zeta(j, k+1)+1-\frac{m+1}{k}-\ln k .
$$

It can be easily shown that

(i) $\Delta h_{R}^{L}\left(m, k, Z_{1}\right)$ is a monotone decreasing in $m$, for each $k>1$.

(ii) $\Delta h_{R}^{U}\left(m, k, Z_{1}\right)$ is a positive function for $m>k \ln k-k-1$ and otherwise is a negative function.

(iii) $\Delta h_{R}^{L}\left(m, k, Z_{1}\right)<2\left(\psi(k)+\gamma^{*}-k \zeta(2, k+1)\right)+1-\ln k, \quad \forall m$.

(see Fig. 5. for illustration.)

Remark 3.3. Note that for $k=1$, i.e. for ordinary records, it is easy to see that the differential entropy has the following properties

(i) $\Delta h_{R}^{U}\left(m, 1, Z_{1}\right)=m+2$ and $\Delta h_{R}^{L}\left(m, 1, Z_{1}\right)=m+2-2 \sum_{j=2}^{m+1} \zeta(j)$.

(ii) The sequence $\left\{h_{R}^{U}\left(m, 1, Z_{1}\right)\right\}$ contains positive and increasing functions in $m$.

(iii) $\Delta h_{R}^{U}\left(m, 1, Z_{1}\right)$ is a positive monotone increasing function of $m$.

(iv) $h_{R}^{U}\left(m, 1, Z_{1}\right)>2$ and $\Delta h_{R}^{U}\left(m, 1, Z_{1}\right)>3$, for each $m$.

(v) $\forall m \geq 7, \quad\left|\Delta h_{R}^{L}\left(m, 1, Z_{1}\right)-(-m-1)\right|<0.01$.

Let

$$
\Delta^{*} h_{R}^{U}(m, k)=h_{R}^{U}(m, k+1)-h_{R}^{U}(m, k)
$$

and

$$
\Delta^{*} h_{R}^{L}(m, k)=h_{R}^{L}(m, k+1)-h_{R}^{L}(m, k) .
$$

It can be shown that

$$
\Delta^{*} h_{R}^{U}\left(m, k, Z_{1}\right)=m \ln \frac{k}{k+1}-\frac{m(m+1)}{2 k(k+1)},
$$


and

$$
\begin{aligned}
\Delta^{*} h_{R}^{L}\left(m, k, Z_{1}\right) & =m\left(\frac{m+1}{2 k(k+1)}+\frac{2}{k+1}+\ln \frac{k}{k+1}\right)+\frac{2}{k} \sum_{j=2}^{m}(m-j+1)\left(\frac{k}{k+1}\right)^{j} \\
& +2 \sum_{j=2}^{m}(m-j+1)\left(k^{j-1}-(k+1)^{j-1}\right) \zeta(j, k+2) .
\end{aligned}
$$

Therefore

(i) $\Delta^{*} h_{R}^{U}\left(m, k, Z_{1}\right)$ is a negative increasing function of $k$ for each $m$. Hence $h_{R}^{U}\left(m, k, Z_{1}\right)$ is a decreasing function of $k$ for each $m$.

(ii) $\lim _{k \rightarrow \infty} \Delta^{*} h_{R}^{U}\left(m, k, Z_{1}\right)=0$ and $-m\left(\ln 2+\frac{m+1}{4}\right)<\Delta^{*} h_{R}^{U}\left(m, k, Z_{1}\right)<0$.

Theorem 3.4. The SI contained respectively in the upper and lower k-record values and associated $k$-record times for $k>1$ are obtained as

$$
\begin{aligned}
h_{R T}^{U}\left(m, k, Z_{1}\right) & =-m \ln k+\frac{m}{k}(m+k)+\left(1-\gamma^{*}-\psi(k+1)\right)\left(m+k-1-\frac{k^{m}}{(k-1)^{m-1}}\right) \\
& +\sum_{j=2}^{m-1}\left((m+k-j) k^{j-1}-\frac{k^{m}}{(k-1)^{m-j}}\right) \zeta(j, k+1),
\end{aligned}
$$

and

$$
\begin{aligned}
h_{R T}^{L}\left(m, k, Z_{1}\right) & =-m \ln k+\frac{m(k-1)}{k}+\left(1-\gamma^{*}-\psi(k+1)\right)\left(m+k-1-\frac{k^{m}}{(k-1)^{m-1}}\right) \\
& +2 m\left(\psi(k+1)+\gamma^{*}\right)+\sum_{j=2}^{m-1}\left((k+j-m-2) k^{j-1}-\frac{k^{m}}{(k-1)^{m-j}}\right) \zeta(j, k+1) \\
& -2 k^{m-1} \zeta(m, k+1) .
\end{aligned}
$$

Remark 3.4. Note that for $k=1$, (3.13) and (3.14) lead to

$$
h_{R T}^{U}\left(m, 1, Z_{1}\right)=\frac{m(3 m+1)}{2}-\sum_{j=2}^{m}(m-j+1) \zeta(j),
$$

and

$$
h_{R T}^{L}\left(m, 1, Z_{1}\right)=m^{2}+m-3 \sum_{j=2}^{m}(m-j+1) \zeta(j) .
$$

Corollary 3.7. For $Z_{\alpha}$, we have

$$
h_{R T}^{U}\left(m, k, Z_{\alpha}\right)=h_{R T}^{U}\left(m, k, Z_{1}\right)-m \ln \alpha+\left(\frac{1}{\alpha}-1\right) \frac{m(m+1)}{2 k},
$$

and

$$
\begin{aligned}
h_{R T}^{L}\left(m, k, Z_{\alpha}\right) & =h_{R T}^{L}\left(m, k, Z_{1}\right)-m \ln \alpha+m\left(\frac{1}{\alpha}-1\right)\left(\psi(k+1)+\gamma^{*}\right) \\
& +\left(1-\frac{1}{\alpha}\right) \sum_{j=2}^{m}(m-j+1) k^{j-1} \zeta(j, k+1),
\end{aligned}
$$


and for $k=1$,

$$
h_{R T}^{U}\left(m, 1, Z_{\alpha}\right)=h_{R T}^{U}\left(m, 1, Z_{1}\right)-m \ln \alpha+\left(\frac{1}{\alpha}-1\right) \frac{m(m-1)}{2},
$$

and

$$
h_{R T}^{L}\left(m, 1, Z_{\alpha}\right)=h_{R T}^{L}\left(m, 1, Z_{1}\right)-m \ln \alpha+\left(\frac{1}{\alpha}-1\right) \frac{m(m-1)}{2}-\left(\frac{1}{\alpha}-1\right) \sum_{j=2}^{m-1}(m-j) \zeta(j) .
$$

Corollary 3.8. For the Pareto (IV), we have

$$
h_{R T}^{U}(m, k, X)=h_{R T}^{U}\left(m, k, Z_{\alpha}\right)+m \ln (\theta \gamma)-\frac{m(m+1)(1-\gamma)}{2 k \alpha}+(1-\gamma) \sum_{j=1}^{m} \sum_{q=k \alpha+1}^{\infty} \frac{(k \alpha)^{j}}{(q-k \alpha) q^{j}},
$$

and

$$
\begin{aligned}
h_{R T}^{L}(m, k, X)= & h_{R T}^{L}\left(m, k, Z_{\alpha}\right)+m \ln (\theta \gamma)+\frac{m(1-\gamma)}{\alpha}\left(\psi(k+1)+\gamma^{*}\right)-\frac{(1-\gamma)}{\alpha} \sum_{j=2}^{m}(m-j+1) \\
& k^{j-1} \zeta(j, k+1)+(1-\gamma) \sum_{j=1}^{m} \sum_{l=1}^{\infty} \sum_{q=0}^{\infty}\left(\begin{array}{c}
\frac{l}{\alpha} \\
q
\end{array}\right) \frac{(-1)^{q} k^{j}}{l(q+k)^{j}} .
\end{aligned}
$$

When $\alpha$ is integer,

$$
\begin{aligned}
h_{R T}^{U}(m, k, X)= & h_{R T}^{U}\left(m, k, Z_{\alpha}\right)+m \ln (\theta \gamma)+m(1-\gamma)\left(\psi(k \alpha+1)+\gamma^{*}\right)-(1-\gamma) \\
& \sum_{j=2}^{m}(m-j+1)(k \alpha)^{j-1} \zeta(j, k \alpha+1)-\frac{m(m+1)(1-\gamma)}{2 k \alpha} .
\end{aligned}
$$

We note that for ordinary records,

$$
\begin{aligned}
h_{R T}^{U}(m, 1, X)= & h_{R T}^{U}\left(m, 1, Z_{\alpha}\right)+m \ln (\theta \gamma)+(1-\gamma) \sum_{i=1}^{m} \sum_{j=\alpha+1}^{\infty} \frac{\alpha^{i}}{(j-\alpha) j^{i}}-\frac{m(m+1)(1-\gamma)}{2 \alpha} \\
h_{R T}^{L}(m, 1, X) & =h_{R T}^{L}\left(m, 1, Z_{\alpha}\right)+m \ln (\theta \gamma)-\frac{m(m+1)(1-\gamma)}{2 \alpha}+\frac{1-\gamma}{\alpha} \sum_{j=2}^{m}(m-j+1) \zeta(j) \\
& +(1-\gamma) \sum_{i=1}^{m} \sum_{j=1}^{\infty} \sum_{q=0}^{\infty}\left(\begin{array}{c}
\frac{j}{\alpha} \\
q
\end{array}\right) \frac{(-1)^{q}}{j(q+1)^{i}}
\end{aligned}
$$

and when $\alpha$ is integer

$$
\begin{aligned}
h_{R T}^{U}(m, 1, X)= & h_{R T}^{U}\left(m, 1, Z_{\alpha}\right)+m \ln (\theta \gamma)+m(1-\gamma)\left(\psi(\alpha+1)+\gamma^{*}\right)-(1-\gamma) \\
& \sum_{j=2}^{m}(m-j+1) \alpha^{j-1} \zeta(j, k+1)-\frac{m(m+1)(1-\gamma)}{2 \alpha} .
\end{aligned}
$$

Let

$$
\Delta h_{R T}^{U}(m, k)=h_{R T}^{U}(m+1, k)-h_{R T}^{U}(m, k),
$$

and 

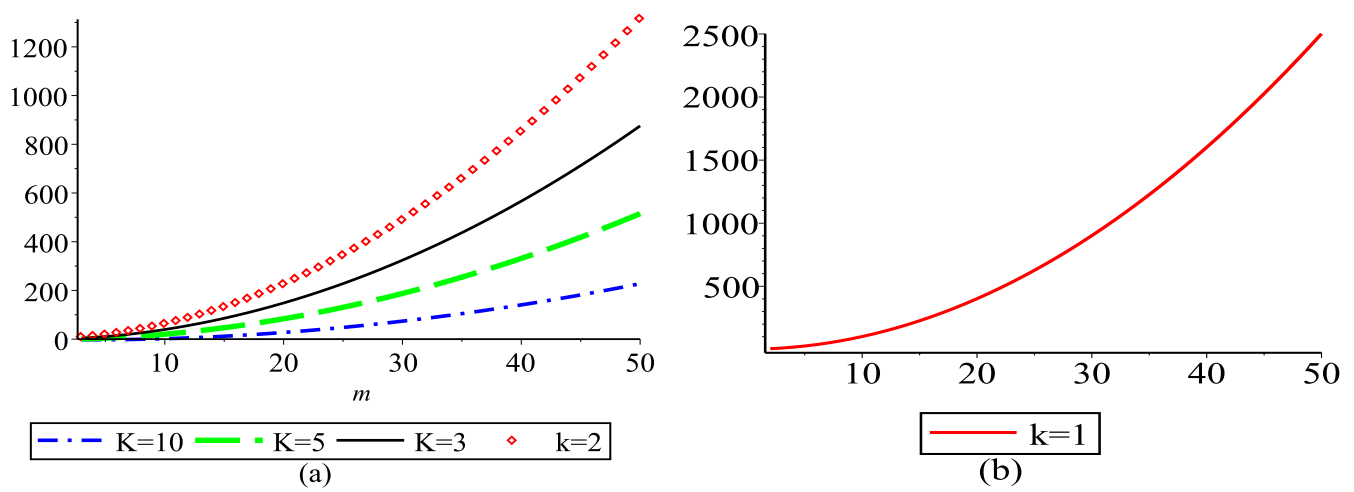

Fig. 6. (a) and (b) represent $h_{R T}^{U}\left(m, k, Z_{1}\right)$ and $h_{R T}^{U}\left(m, 1, Z_{1}\right)$, respectively.

$$
\Delta h_{R T}^{L}(m, k)=h_{R T}^{L}(m+1, k)-h_{R T}^{L}(m, k) .
$$

Then,

$$
\begin{aligned}
\Delta h_{R T}^{U}\left(m, k, Z_{1}\right) & =-\ln k+\frac{2 m+1}{k}+1+\left(1-\gamma^{*}-\psi(k+1)\right)\left(1-\frac{k^{m}}{(k-1)^{m}}\right) \\
& +\sum_{j=2}^{m-1}\left(k^{j-1}-\frac{k^{m}}{(k-1)^{m-j+1}}\right) \zeta(j, k+1)-\frac{k^{m-1}}{k-1} \zeta(m, k+1),
\end{aligned}
$$

and

$$
\begin{aligned}
\Delta h_{R T}^{L}\left(m, k, Z_{1}\right)= & -\ln k+\frac{k-1}{k}+\left(1-\gamma^{*}-\psi(k+1)\right)\left(1-\frac{k^{m}}{(k-1)^{m}}\right) \\
+ & 2\left(\psi(k+1)+\gamma^{*}\right)-2 k^{m} \zeta(m+1, k+1)+\frac{k^{m-1}-2 k^{m}}{k-1} \\
& \zeta(m, k+1)-\sum_{j=2}^{m-1}\left(\frac{k^{m}}{(k-1)^{m-j+1}}+k^{j-1}\right) \zeta(j, k+1) .
\end{aligned}
$$

respectively. We note that for $k=1$,

$$
\Delta h_{R T}^{U}\left(m, 1, Z_{1}\right)=3 m+2-\sum_{j=2}^{m+1} \zeta(j)
$$

and

$$
\Delta h_{R T}^{L}\left(m, 1, Z_{1}\right)=2 m+2-3 \sum_{j=2}^{m+1} \zeta(j), m=1,2,3, \ldots
$$

The following properties are easily obtained:

(i) $\Delta h_{R T}^{L}\left(m, 1, Z_{1}\right)$ is a negative function of $m$, therefore $h_{R T}^{L}\left(m, 1, Z_{1}\right)$ is a decreasing function of $m$. Furthermore, $\Delta h_{R T}^{L}\left(m, 1, Z_{1}\right)$ is an increasing function of $m$.

(ii) For each $m \geq 8$, we have $\left|\Delta h_{R T}^{L}\left(m, 1, Z_{1}\right)-(-m-1)\right|<\frac{1}{100}$ and $\mid \Delta h_{R T}^{U}\left(m, 1, Z_{1}\right)-(2 m+$ $1) \mid<\frac{1}{100}$, for each $m \geq 6$.

( see Figs. 6. and 7. for some more notes.) 

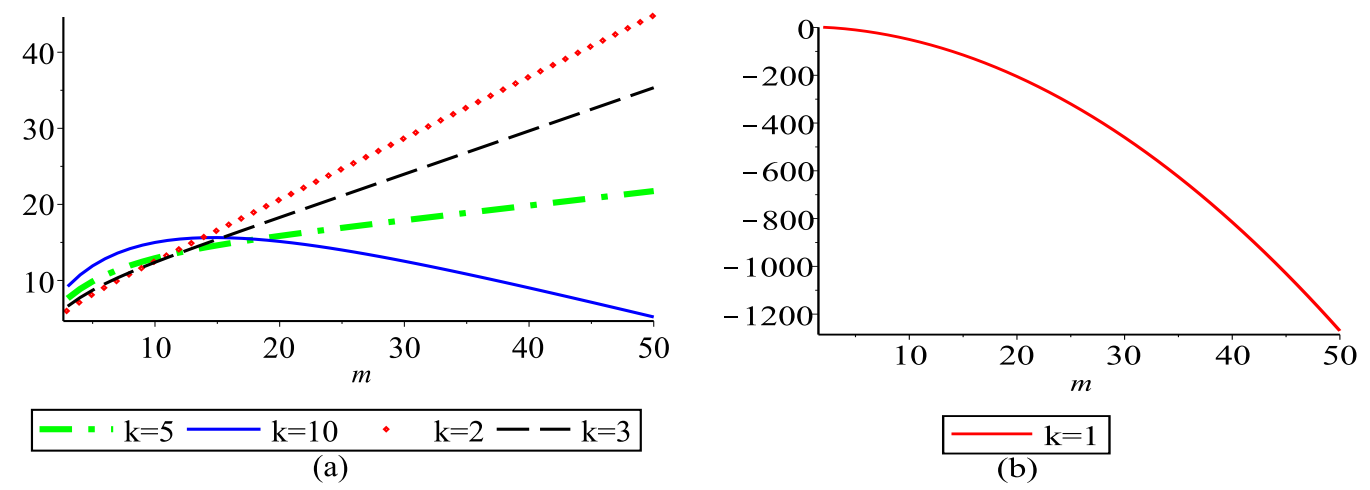

Fig. 7. (a) and (b) represent $h_{R T}^{L}\left(m, k, Z_{1}\right)$ and $h_{R T}^{L}\left(m, 1, Z_{1}\right)$, respectively.

\section{Conclusions}

In this paper, we obtained the Shannon information in $k$-record in a sample of fixed size as well as in an inverse sampling plan for Pareto-type distributions. Properties of entropies for $k$-record values for Pareto-type distributions are also investigated.

\section{Acknowledgments}

The authors are grateful to the editorial board of Journal of Statistical Theory and Applications (JSTA), in particular, Professor Hamedani and would like to thank two anonymous referees for useful comments and suggestions that lead to this improved version of the paper.

\section{References}

[1] B. Afhami and M. Madadi, Shannon entropy in generalized order statistics from Pareto-type distributions, Int. J. Nonlinear. Anal. Appl. 4 (2013) 79-91.

[2] B.C. Arnold, Pareto Distribution, (International Cooperative Publishing House, Fairland, Maryland, 1983).

[3] B.C. Arnold, N. Balakrishnan and H.N. Nagaraja, Records, (John Wiley and Sons, New York, 1998).

[4] S. Baratpour, J. Ahmadi and N. R. Arghami, Entropy properties of record statistics, Statist. Papers 48 (2007) 197-213.

[5] L. Boltzmann, Neitere Studien uber das Warmegleichgewicht unter Gasmolekulen. K. Akad. Wiss. (Wein) Sitzb. 66 (1872) 275.

[6] R. Clausius, Abhaudlungen uber die mechanische Warmetheorie Friedrich, (Vieweg, Braunschweig, 1864).

[7] T.M. Cover and J.A. Thomas, Elements of Information Theory, (Wiley, New York, 2006).

[8] W. Dziubdziela and B. Kopociński, Limiting properties of the $k$-th record values, Appl. Math. 2 (1976) 187-190.

[9] N. Ebrahimi, E. S. Soofi and H. Zahedi, Information properties of order statistics and spacings, IEEE Trans. Inform. Theory 50 (2004) 177-183.

[10] Z. Grudzien and D. Szynal, On the expected values of $k$-th record values and associated characterizations of distributions. Probab. Stat. Decis. Theory Ser. A, Reidel, Dordrecht (1985) 119-127.

[11] M. Madadi and M. Tata, Shannon information in record data, Metrika 74 (2011) 11-31.

[12] M. Madadi and M. Tata, Shannon information in k-records, Comm. Statist. Theory Methods 43 (2014) 3286-3301. 
[13] B.O. Oluyede, Some general notions of stochastic orderings for weighted reliability and uncertainty measures with applications, Int. Math. Forum 1 (2006) 51-60.

[14] S. Park, The entropy of consecutive order statistics, IEEE Trans. Inform. Theory 1 (1995) 2003-2007.

[15] C.E. Shannon, A mathematical theory of communication. Bell Syst. Tech. J.27 (1948) 379-423.

[16] G. H. Yari and G. M. Borzadaran, Entropy for Pareto-types and its order statistics distributions, Commun. Inf. Syst. 10 (2010) 193-202. 